\title{
ESTIMAÇÃO DE DEMANDA ENERGIA ELÉTRICA DE CONTRATO ÓTIMA PARA UPV*
}

\section{Resumo}

Ródnei Abbade $\mathrm{Abreu}^{2}$

Nelson Neves Teixera ${ }^{3}$

Hyndrews Fernandes Staneck ${ }^{4}$

A UPV é abastecida basicamente por duas fontes de energia elétrica de $60 \mathrm{~Hz}$ (maioria das cargas da usina): LIGHT (concessionária - externa) e CTE\#2 (interna). A energia externa chega a UPV via sistema de transmissão e distribuição da LIGHT, por esse transporte paga-se uma tarifa de uso do sistema para a concessionária, vale ressaltar que não é comprado energia da LIGHT e sim o transporte da mesma, a energia é comprada de unidades geradoras distantes da UPV. O valor pago pelo transporte da energia é baseado em um contrato de Demanda [MW], que representa um valor máximo que o sistema deve ser capaz de suportar, caso seja solicitado. 0 objetivo desse trabalho é identificar qual a melhor (menor) demanda a ser contratada pela UPV, de forma a atender os processos produtivos e não ultrapassar o valor contratado (pagamento de multa), para tal foi criado um simulador com base em dados históricos para estimação de demanda a ser contratada pela usina.

Palavras-chave: Contratação de demanda e simulação.

\section{ELECTRIC POWER DEMAND ESTIMATION OF OPTIMAL CONTRACT FOR UPV}

\begin{abstract}
The UPV is fueled primarily by two sources of electricity $60 \mathrm{~Hz}$ (most power plant loads): LIGHT (dealership-external) and CTE2 (internal). External power reaches UPV through the transmission and distribution system of LIGHT, by this transport pays a fee to use the system for the concessionaire, it is worth mentioning that it's not bought energy from LIGHT but your transport, energy is bought from distant generating units of the UPV. The value paid for the energy transport is based on a contract of Demand MW, representing a maximum value that the system must be capable of supporting, if requested. The objective of this work is to identify what the best (lowest) demand to be hired by the UPV, in order to meet the production processes and does not exceed the value hired (payment of fines), was created a simulator based on historical data for estimation of demand being contracted by power plant.
\end{abstract}

Keywords: Hiring demand and simulation.

1 Engenheiro Manutenção, Gerência de Distribuição de Energéticos, Companhia Siderúrgica Nacional, Volta Redonda, RJ, Brasil.

2 Coordenador de Projetos Especiais, Gerência Geral de Energéticos e Utilidades, Companhia Siderúrgica Nacional, Volta Redonda, RJ, Brasil.

3 Técnico de Produção, Gerência de Distribuição de Energéticos, Companhia Siderúrgica Nacional, Volta Redonda, RJ, Brasil. 


\section{INTRODUÇÃO}

A UPV possui uma unidade de geração de energia elétrica (CTE-2) localizada dentro da usina que, em condições normais (operação econômica), supre aproximadamente $40 \%$ de sua necessidade de energia elétrica. Como consumidor livre (Resolução ANEEL no. 264 (13/08/1998)), a CSN pode escolher no mercado, e a seu inteiro critério, o fornecedor de energia elétrica para fornecimento da energia faltante para atender a seus processos.

Como a energia elétrica mais barata encontra-se em usinas de geração distantes da UPV, além da compra da energia, é preciso pagar pelo transporte da mesma até a unidade consumidora. Para isso a UPV mantém um contrato de uso do sistema de distribuição com a concessionária Light, pagando mensalmente parcela fixa correspondente aos valores contratados de demanda. Em caso de a demanda máxima registrada no mês ultrapassar o valor contratado mais cinco por cento, o montante ultrapassado é faturado com tarifa três vezes maior que a normal. Para evitar pagamento de multas por ultrapassagem da demanda contratada, a UPV dispõe de sistema para controle gerenciado pelo Sistema de Gerenciamento de Energia (SIGEN), que sinaliza para determinadas áreas de produção (denominadas áreas controláveis) as necessidades de redução ou parada em função de eventual projeção de ultrapassagem.

O objetivo do trabalho é auxiliar na especificação da demanda de contrato de energia elétrica, de forma a reduzir os custos nessa contratação para Usina Presidente Vargas (UPV) e, ao mesmo tempo, garantir atendimento às necessidades das áreas de produção em termos de energia elétrica.

\subsection{Definições para a Contratação de Demanda}

A Contratação é regida basicamente pela Resolução ANEEL № 414 (09/09/10), abaixo são apresentadas algumas definições da resolução que ajudará no entendimento da Contratação de Demanda no que compete de importante ao contrato da UPV.

Capítulo 1 - Definições (Resolução ANEEL № 414):

Item XX1 - Demanda Contratada: "demanda de potência ativa a ser obrigatória e continuamente disponibilizada pela distribuidora, no ponto de entrega, conforme valor e período de vigência fixados em contrato, e que deve ser integralmente paga, seja ou não utilizada durante o período de faturamento, expressa em quilowatts $(\mathrm{kW}) ; "$

Item LXXV - Tarfia: "valor monetário estabelecido pela ANEEL, fixado em R\$ (Reais) por unidade de energia elétrica ativa ou da demanda de potência ativa, sendo:

- tarifa de uso do sistema de distribuição - TUSD: valor monetário unitário determinado pela $A N E E L$, em $R \$ / M W h$ ou em $R \$ / k W$, utilizado para efetuar o faturamento mensal de usuários do sistema de distribuição de energia elétrica pelo uso do sistema."

\subsubsection{Seção II - Da Ultrapassagem}

Art. 93. "Quando os montantes de demanda de potência ativa ou de uso do sistema de distribuição - MUSD medidos excederem em mais de $5 \%$ (cinco por cento) os valores contratados, deve ser adicionada ao faturamento regular a cobrança pela ultrapassagem conforme a seguinte equação:

$\operatorname{Dultrapassagem}(p)=[\operatorname{PAM}(p)-\operatorname{PAC}(p)] \times 2 \times \operatorname{VRdult}(p)$ 


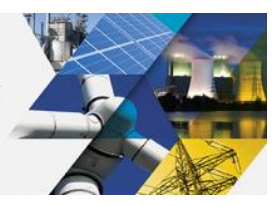

onde:

Dultrapassagem $(p)$ = valor correspondente à demanda de potência ativa ou MUSD excedente, por posto tarifário "p", quando cabível, em Reais (R\$);

- $\operatorname{PAM}(p)=$ demanda de potência ativa ou MUSD medidos, em cada posto tarifário "p" no período de faturamento, quando cabível, em quilowatt (kW);

- $\operatorname{PAC}(p)=$ demanda de potência ativa ou MUSD contratados, por posto tarifário "p" no período de faturamento, quando cabível, em quilowatt $(\mathrm{kW})$;

- $\operatorname{VR} \operatorname{dult}(p)$ = valor de referência equivalente às tarifas de demanda de potência aplicáveis aos subgrupos do grupo A ou as TUSD-Consumidores-Livres; e

- $\mathrm{p}=$ indica posto tarifário ponta ou fora de ponta para as modalidades tarifárias horárias ou período de faturamento para a modalidade tarifária convencional binômia."

Além dessas definições, é importante definir outros dois itens também estabelecidos por esta resolução e definidos pela empresa distribuidora, no caso da UPV a LIGHT, que são estes:

- Horário de Ponta: "O horário de ponta para o consumo de energia elétrica é o período do dia de maior utilização de rede da Light. Corresponde ao período entre 17 h30 e 20h30, com exceção de sábados, domingos e feriados nacionais."

- Horário Fora de Ponta: "Período composto pelo conjunto das horas diárias consecutivas e complementares àquelas definidas nos postos ponta."

A definição de Horário de Ponta e Fora de Ponta é importante, pois a tarifa nos dois horários são diferentes, aproximadamente 4 vezes maior no Horário de Ponta e além disso contrata-se Demanda, iguais ou não, para os dois Horários.

\section{HISTÓRICO}

Na Figura 1 é possível observar as demandas máximas atingidas em cada mês pela UPV, considerando o período de Janeiro de 2010 a Dezembro de 2012.

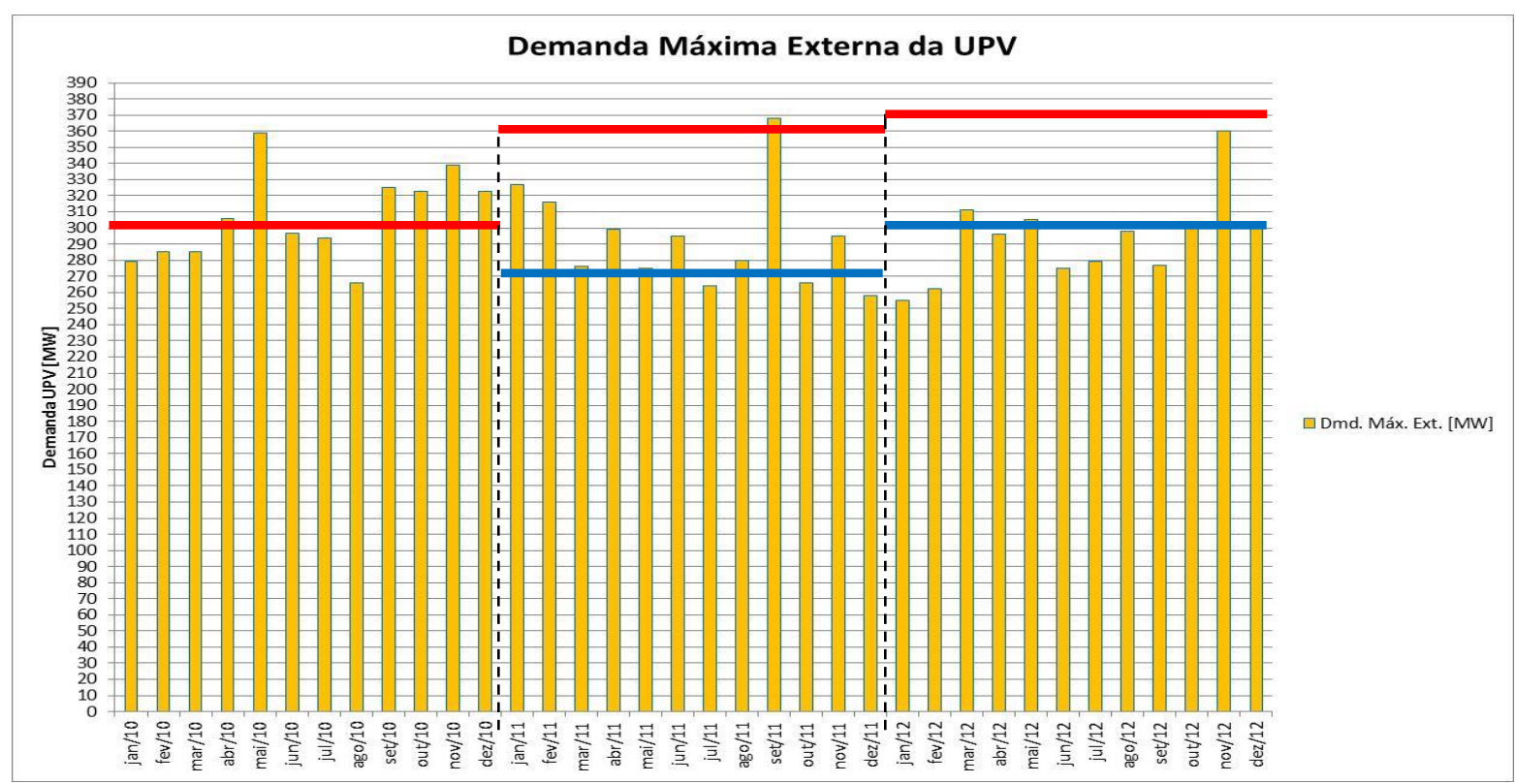

Figura 1 - Histórico de demanda externa da UPV.

No gráfico da Figura 1 são notados também os limites de demanda que era possível se atingir, sem pagamento de multa por ultrapassagem, com o contrato de demanda 


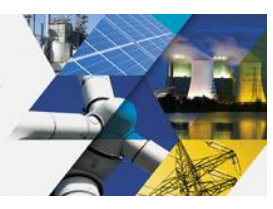

firmado com a concessionária em cada período. Uma observação importante desses limites é que durante o ano de 2010 tinha-se contratado uma demanda de $310 \mathrm{MW}$, no ano de 2011 uma demanda de 280 MW mais uma Reserva de Capacidade de 90 MW e em 2012 uma demanda de 310 somada a uma Reserva de Capacidade de 70 MW.

A título de conhecimento e entendimento, a Reserva de Capacidade é definida pela resolução ANEEL 304 de 04/03/08 como:

"Reserva de capacidade é o montante de uso, em MW, requerido dos sistemas elétricos de transmissão ou de distribuição para suprimento a uma ou mais unidades consumidoras diretamente conectadas à usina de autoprodutor de energia, quando da ocorrência de interrupções ou reduções temporárias na geração de energia elétrica da referida usina, adicionalmente ao montante de uso já contratado de forma permanente para atendimento às referidas unidades consumidoras"

A forma de pagamento do montante contratado de reserva de capacidade dá-se de acordo com a fórmula:

Onde:

$$
E_{R C}=\left(M_{p} x T_{p}+M_{f p} x T_{f p}\right) x \frac{n_{u}}{n_{m}}
$$

$E_{\mathrm{RC}}$ : Encargo mensal pelo uso da reserva de capacidade, em $\mathrm{R} \$$;

nu: Número de dias em que houve utilização da reserva de capacidade no mês em referência;

$\mathrm{n}_{\mathrm{m}}$ : Número de dias do mês em referência;

$\mathrm{T}_{\mathrm{p}}$ : Tarifa de uso do sistema de distribuição no horário de ponta para unidades consumidoras, em $\mathrm{R} \$ / \mathrm{kW}$;

$\mathrm{T}_{\mathrm{fp}}$ : Tarifa de uso do sistema de distribuição no horário fora de ponta para unidades consumidoras, em $\mathrm{R} \$ / \mathrm{kW}$;

$\mathrm{Mp}$ : Montante de uso de reserva de capacidade para o horário de ponta, em kW maior valor entre o contratado e o verificado por medição no mês em referência, devendo o referido valor contratado ser único para todo ciclo contratual;

Mfp: Montante de uso de reserva de capacidade para o horário fora de ponta, em kW - maior valor entre o contratado e o verificado por medição no mês em referência, devendo o referido valor contratado ser único para todo o ciclo contratual.

Nota-se que a reserva de capacidade, que só pode ser utilizadas 60 dias no ano, é um excelente negócio para empresas com unidades de geração instaladas em suas plantas, que é o caso da UPV. Entretanto, por questões de dificuldades contratuais e por capacidade do sistema LIGHT, a UPV não dispõe mais do recurso da Reserva de capacidade, agora no ano de 2013.

Além destes gastos, com contratação de demanda, que quando consideramos o valor de $310 \mathrm{MW}$, chegam a aproximadamente $\mathrm{R} \$ 12.000 .000,00$ / mês, foi verificado, em 2010, histórico de registros de interferências em áreas de produção por controle de demanda (tabela 1). 


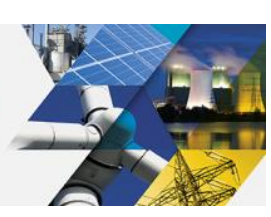

Tabela 1 - Histórico interferências (tempo em minutos)

\begin{tabular}{|l|l|r|}
\hline Ano & Equipamento (PE) & Tempo \\
\hline \multirow{2}{*}{2010} & LTF1 & 170 \\
& LTF3 & 293 \\
& LTQ2 & 1035 \\
\hline 2010 Total & 1498 \\
\hline \multicolumn{2}{|l|}{ Total geral } & 7815 \\
\hline
\end{tabular}

É possível perceber pelo gráfico da Figura 1 que, desde quando a UPV optou pela contratação da Reserva de Capacidade, não foi percebido problemas com ultrapassagem de demanda. Observa-se que apenas em Setembro de 2011 e Novembro de 2012 a usina demandou mais do sistema LIGHT em comparação com todos os outros meses, mas isso é perfeitamente justificável, haja vista que nesses meses aconteceu o "Paradão" da CTE\#2.

Como o contrato de Reserva de Capacidade não mais existe e a UPV tem que se adequar ao novo cenário, esse trabalho vem para auxiliar na contratação da demanda que melhor atenda a usina nesse e em momentos futuros.

\section{DESENVOLVIMENTO}

Com base nas resoluções pertinentes da ANEEL, no histórico de demanda da usina nos últimos 3 anos, nas programações de manutenção na CTE\#2, no conceito de geração econômica na CTE\#2 e na entrada de novas cargas, foi desenvolvido um simulador para identificar a melhor demanda a ser contratada de forma a minimizarmos os custos anuais com fatura de energia elétrica LIGHT, mantendo o atendimento pleno aos clientes da UPV, ou seja, menor custo sem utilização do Controle de Demanda.

\subsection{Histórico de Demanda UPV e Estimação de Demanda Futura}

O desenvolvimento do projeto se inicia com o levantamento do histórico de demanda da usina em operação normal, nota-se que o essencial é a demanda como um todo da usina, ou seja, demanda externa somada a demanda da CTE\#2, para que seja possível entender o comportamento como um todo da UPV. Abaixo (Figura 2) é apresentado esse comportamento. 

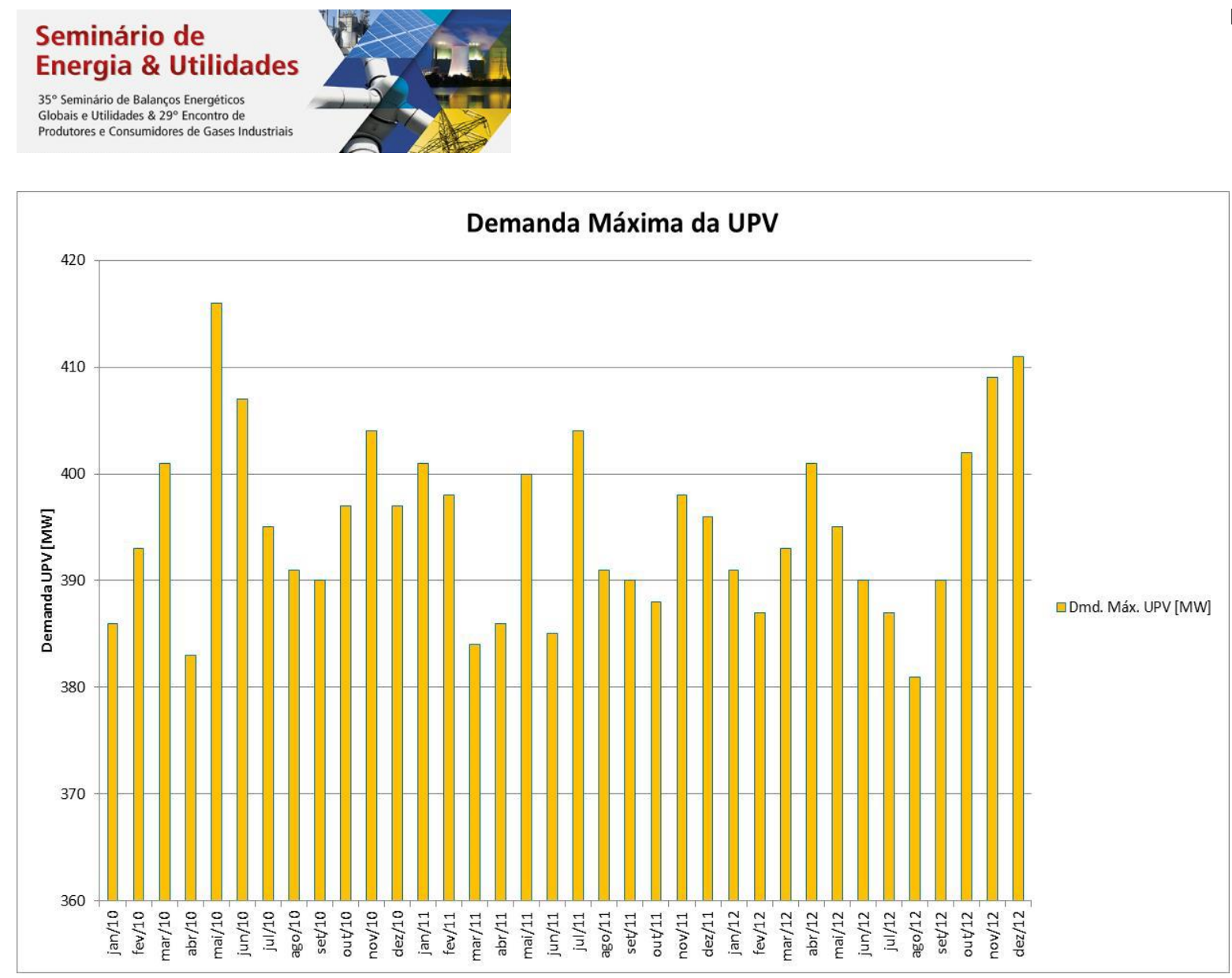

Figura 2 - Demanda Máxima UPV.

Como é possível perceber a demanda da usina tem um comportamento bem aleatório ao longo dos meses e dos anos. Demanda mínima observada de $381 \mathrm{MW}$ e máxima de $416 \mathrm{MW}$. Para estimar uma demanda futura para usina (2013), de forma conservadora, foram selecionadas as maiores demandas de cada mês considerando os 3 últimos anos (2010, 2011 e 2012), o resultado de estimação é apresentado na Figura 3.

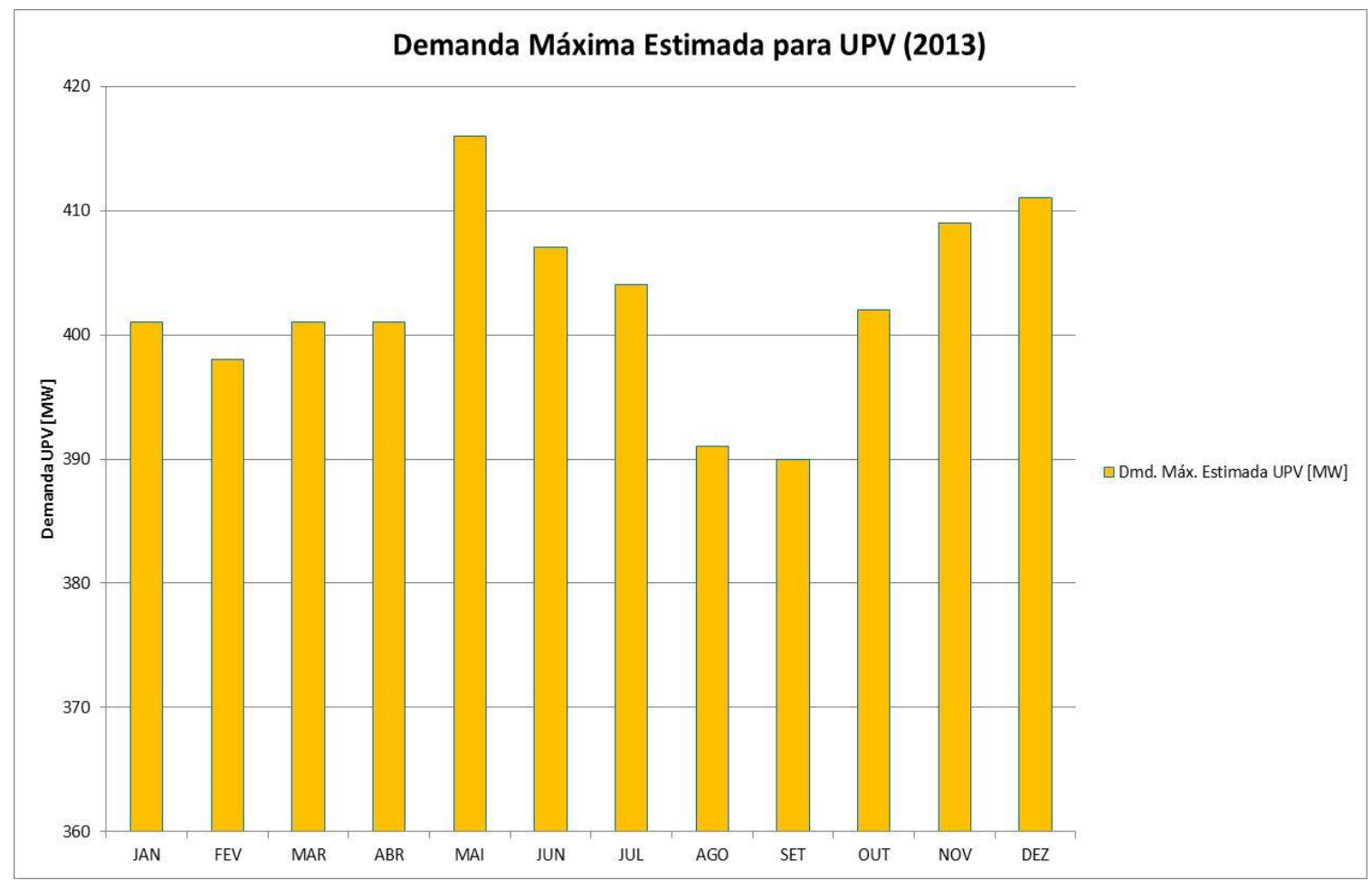

Figura 3 - Demanda estimada para UPV em 2013.

* Contribuição técnica ao $35^{\circ}$ Seminário de Balanços Energéticos Globais e Utilidades e $29^{\circ}$ Encontro de Produtores e Consumidores de Gases Industriais, 13 a 15 de agosto de 2014, São Paulo, SP, Brasil. 


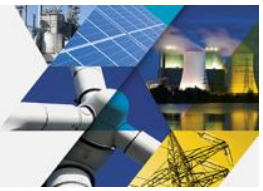

\subsection{Programação de Paradas da CTE\#2}

Como visto anteriormente, o momento mais crítico, em termos de demanda para UPV, são as paradas da CTE\#2, então foram levantadas as programações de paradas dos equipamentos da CTE\#2 que influenciam em nível de geração, que, no caso, são as caldeiras e turbo-geradores, na tabela a seguir (Tabela 2), são mostradas as programações para 2013, com o nível de geração esperado para cada ocasião.

Tabela 2 - Programação de paradas na CTE\#2

Programação de Paradas - GGE 2013

\begin{tabular}{|c|c|c|c|c|c|c|c|c|c|c|c|c|c|c|c|c|c|c|c|c|c|c|c|c|}
\hline \multirow[t]{3}{*}{ Equipamento } & \multicolumn{2}{|c|}{ jan } & \multicolumn{2}{|c|}{ fev } & \multicolumn{2}{|c|}{ mar } & \multicolumn{2}{|c|}{$a b r$} & \multicolumn{2}{|c|}{ mai } & \multicolumn{4}{|c|}{\begin{tabular}{l|l} 
jun & jul \\
\end{tabular}} & \multicolumn{2}{|c|}{ ago } & \multicolumn{2}{|c|}{ set } & \multicolumn{2}{|c|}{ out } & \multicolumn{2}{|c|}{ nov } & \multicolumn{2}{|c|}{ dez } \\
\hline & & & & & & & & & & & & PLAN & EJAD & & & & & & & & & & & \\
\hline & \begin{tabular}{|l|}
$N^{\circ}$ \\
dias
\end{tabular} & \begin{tabular}{|l} 
Ger. \\
Méd.M \\
w
\end{tabular} & & 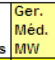 & \begin{tabular}{|l}
$N^{\circ}$ \\
dias
\end{tabular} & $\begin{array}{l}\text { Ger. } \\
\text { Med.M } \\
\text { ww }\end{array}$ & \begin{tabular}{|l} 
No \\
dias
\end{tabular} & $\begin{array}{l}\text { Ger. } \\
\text { Mé.m } \\
\text { w }\end{array}$ & $\begin{array}{l}\text { No } \\
\text { dias }\end{array}$ & $\begin{array}{l}\text { Ger. } \\
\text { Mèd. } \\
\text { Mw }\end{array}$ & \begin{tabular}{|l}
$N^{\circ}$ \\
dias
\end{tabular} & $\begin{array}{l}\text { Ger. } \\
\text { Méd. } \\
\text { mw }\end{array}$ & \begin{tabular}{|l} 
No \\
dias
\end{tabular} & $\begin{array}{l}\text { Ger. } \\
\text { Mad. } \\
\text { Mw }\end{array}$ & \begin{tabular}{|l|l} 
No \\
dias
\end{tabular} & $\begin{array}{l}\text { Ger. } \\
\text { Med. } \\
\text { MW }\end{array}$ & \begin{tabular}{|l} 
No \\
dias
\end{tabular} & \begin{tabular}{|l} 
Ger. \\
Mèd. \\
MWN
\end{tabular} & \begin{tabular}{|l} 
No \\
dias \\
\end{tabular} & $\begin{array}{l}\text { Ger. } \\
\text { Med. } \\
\text { MW }\end{array}$ & \begin{tabular}{|l|}
$N^{\circ}$ \\
dias
\end{tabular} & $\begin{array}{l}\text { Ger. } \\
\text { Méd. } \\
\text { MW }\end{array}$ & \begin{tabular}{|l|}
$w^{\circ}$ \\
dias
\end{tabular} & \begin{tabular}{|c} 
Ger. \\
Mé. \\
Mw
\end{tabular} \\
\hline CTE-2 & & & & & & & & & & & & & & & & & & & & & & & & \\
\hline Caldeira 81 & 2,5 & 100 & & & & & & & & & & & & & & & 30 & 100 & 30 & 100 & & & & \\
\hline Caldeira 82 & & & & & 2,5 & 100 & & & & & & & 15 & 100 & & & & & & & & & & \\
\hline Caldeira 83 & & & & & & & & & 2,5 & 100 & & & & & & & & & & & 30 & 100 & 30 & 100 \\
\hline Parada Total - CTE-2 & & & & & & & & & & & & & & & & & & & & & 5 & 0 & & \\
\hline TGE10 & 2,5 & 90 & & & & & & & & & & & 2,5 & 90 & & & & & & & & & & \\
\hline TGE20 & & & & & 2,5 & 90 & & & & & & & & & & & 2,5 & 90 & & & & & & \\
\hline
\end{tabular}

\subsection{Geração Econômica da CTE\#2}

A CTE\#2 vem a algum tempo trabalhando no modo econômico de geração, o que garante uma geração de aproximadamente 140 MW. A geração econômica é um modo de operação que consiste na utilização mínima de gás natural e aproveitamento máximo do gás siderúrgico barateando o custo operacional da planta. Logo, considerando tal modo de operação, têm-se uma geração de $140 \mathrm{MW}$, exceto, é claro, nos momentos de paradas de equipamentos.

\subsection{Entrada de Novas Cargas}

No trabalho foi considerado a entrada parcial da planta de Aços Longos a partir de Setembro de 2013.

\subsection{O Simulador}

O simulador foi desenvolvido dentro do software Microsoft Excel, que está disponível na maioria das máquinas da usina e é de domínio / uso da grande maioria de pessoas da área. A linguagem de programação utilizada no desenvolvimento foi o VB (Visual Basic), que está disponível no Excel.

$\mathrm{Na}$ Figura 4, é apresentado o algoritmo básico do simulador: 


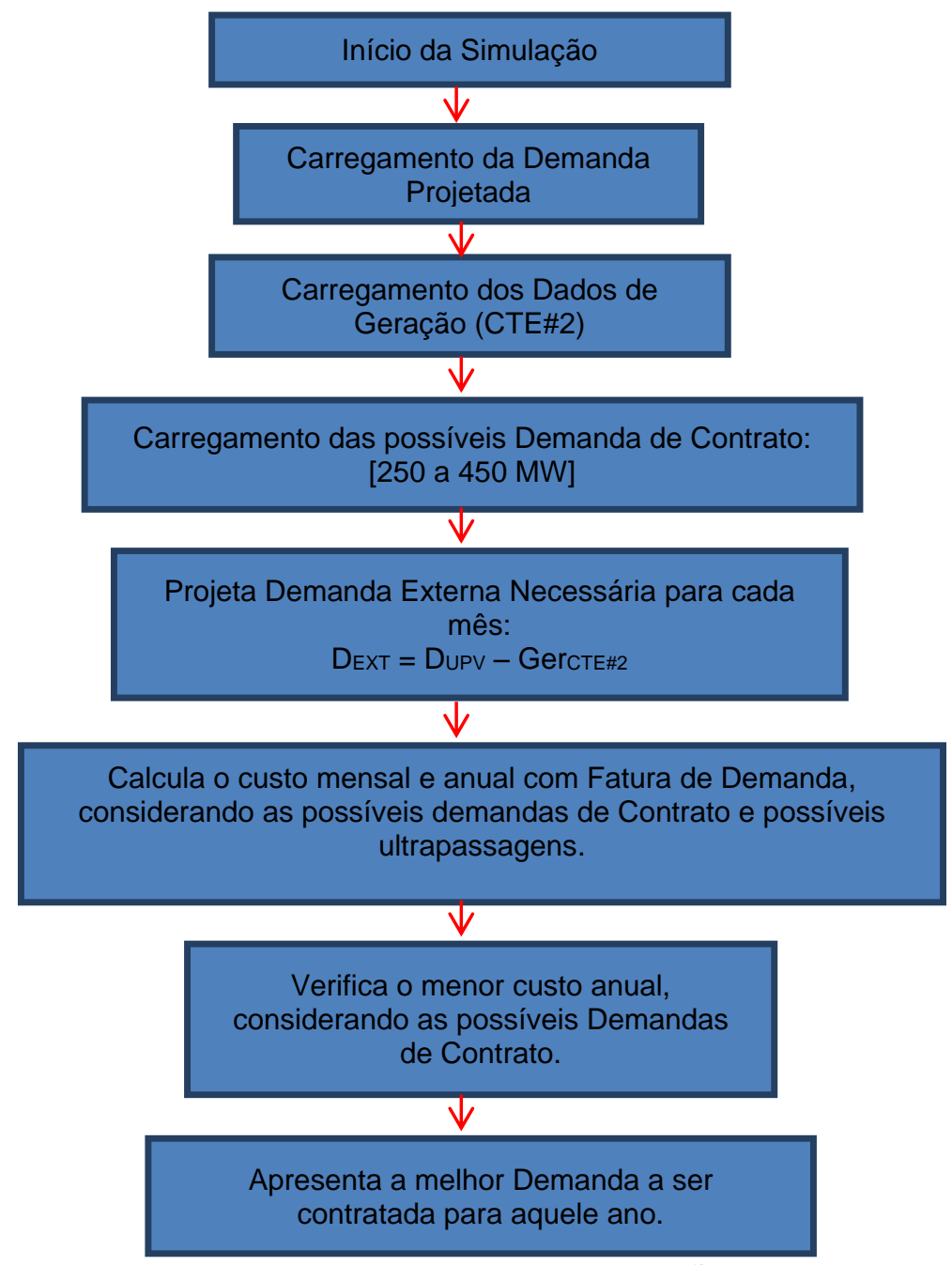

Figura 4 - Algoritmo de simulação.

Na Figura 5 é mostrado parte do programa e seu ambiente de desenvolvimento.

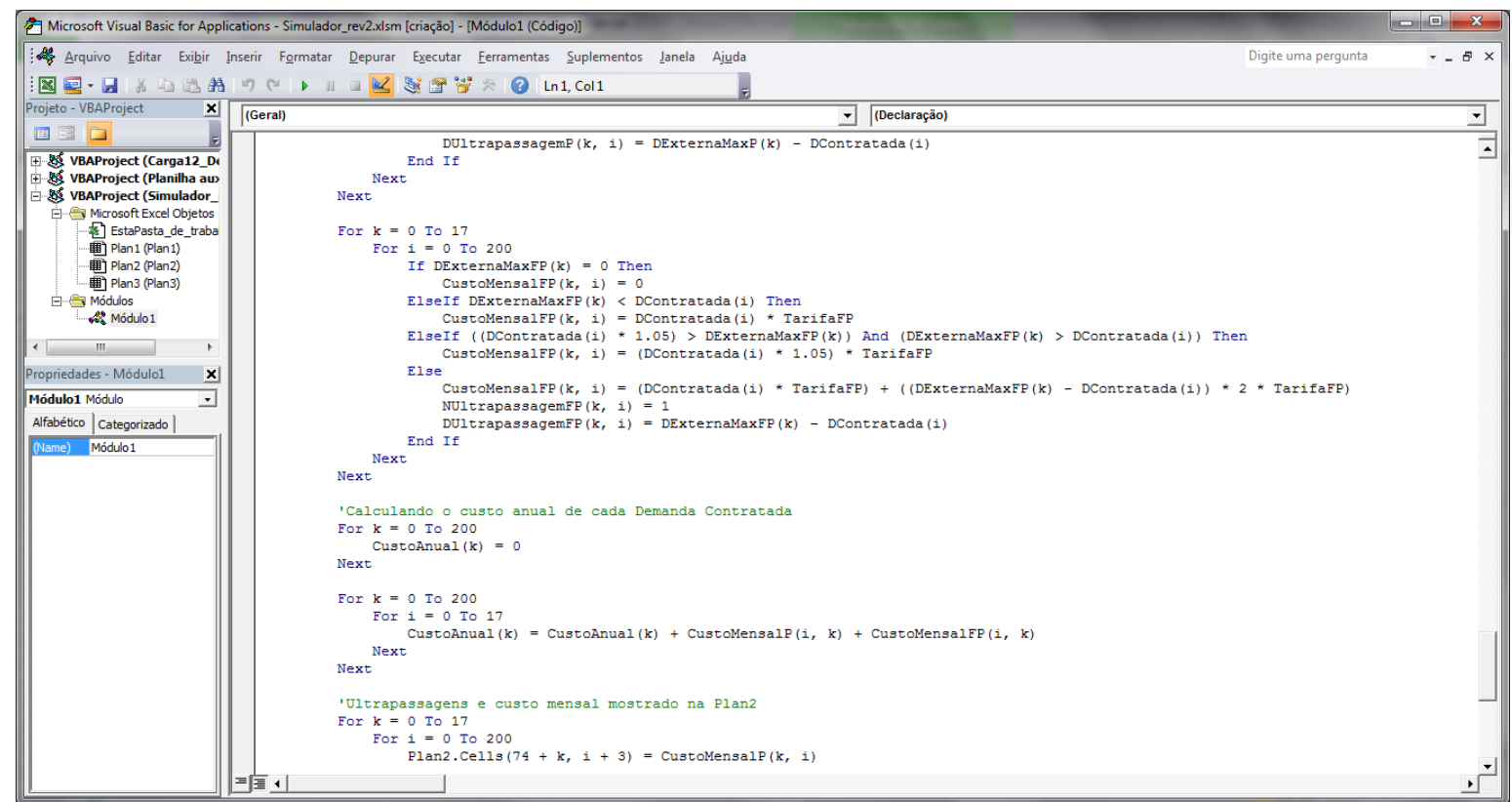

Figura 5 - Ambiente de programação do simulador.

* Contribuição técnica ao $35^{\circ}$ Seminário de Balanços Energéticos Globais e Utilidades e $29^{\circ}$ Encontro de Produtores e Consumidores de Gases Industriais, 13 a 15 de agosto de 2014, São Paulo, SP, Brasil. 


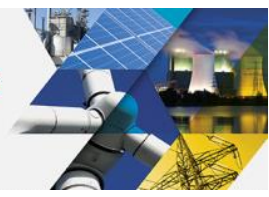

\section{RESULTADOS DAS SIMULAÇÕES}

Diante das regras expostas pelas resoluções da ANEEL e pelas considerações apresentadas no Desenvolvimento foi executada a simulação de demanda a ser contratada para o ano de 2013, o resultado dessa simulação é apresentado na Figura 6.

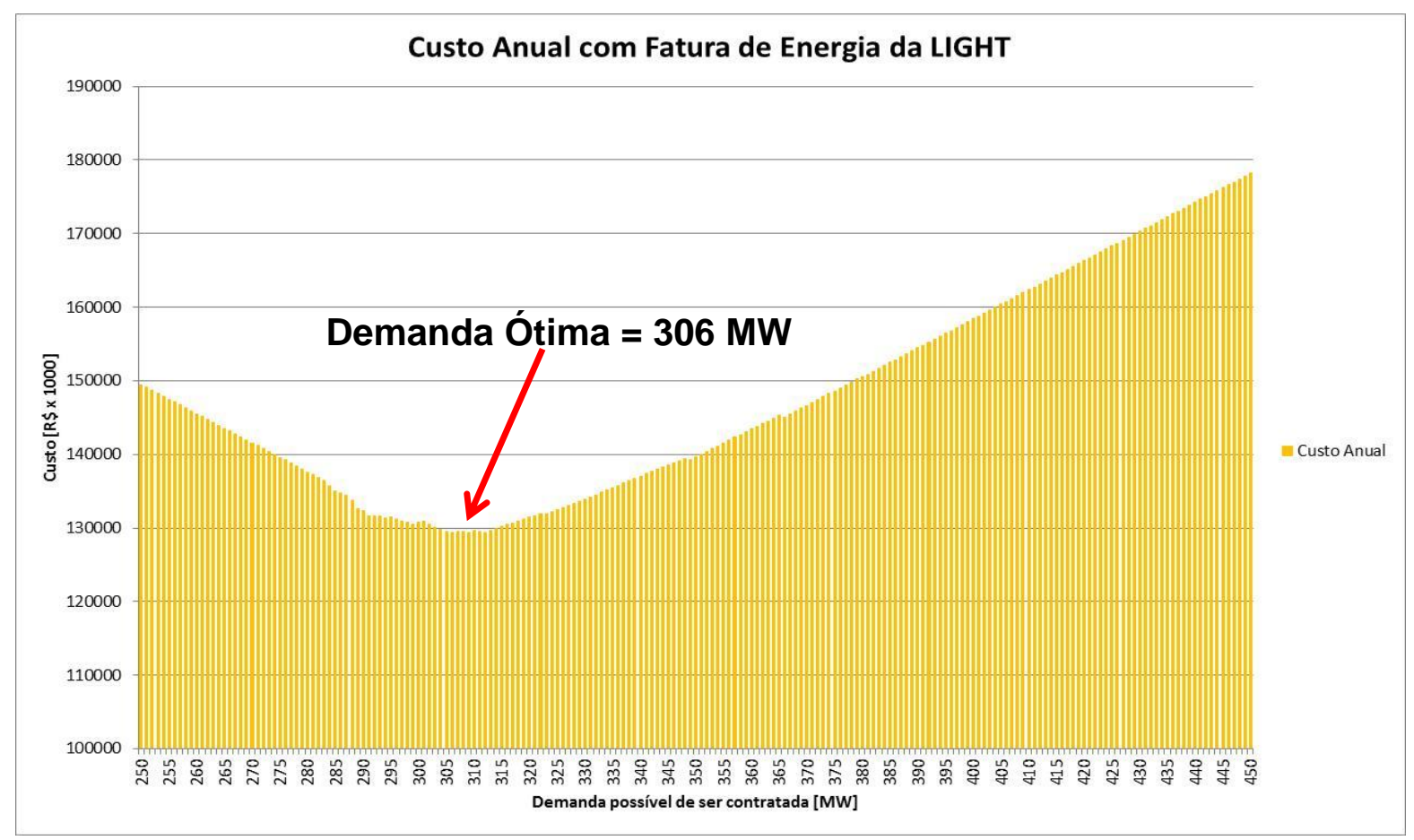

Figura 6 - Gráfico de Demanda a ser contratada.

Como é possível perceber, o simulador indica, para o ano de 2013, uma demanda a ser contratada com a concessionária (LIGHT) de $306 \mathrm{MW}$ com um custo estimado de $\mathrm{R} \$ 129.321 .000,00$ no ano.

\section{IMPLANTAÇÃO}

A CSN no ano de 2013 passa por um momento complicado em relação à concessionária LIGHT devido a problemas com disponibilidade no sistema da LIGHT, atraso no projeto da SE de $500 \mathrm{kV}$ para atendimento a UPV, brigas judiciais, etc. Até o mês de Agosto ainda não foi firmado um contrato para os próximos anos com a LIGHT, hoje a situação da demanda encontra-se com um valor de $310 \mathrm{MW}$ sem Reserva de Capacidade, que nada mais é que o contrato de $2012 \mathrm{com}$ a Reserva de Capacidade cortada pela concessionária.

Como não foi possível aplicar na prática o resultado do simulador, o resultado (Figura 7), aqui apresentado, mostra como estaria a situação com gastos de fatura de energia LIGHT caso os 306 MW tivesse sido contratado em Janeiro de 2013. 

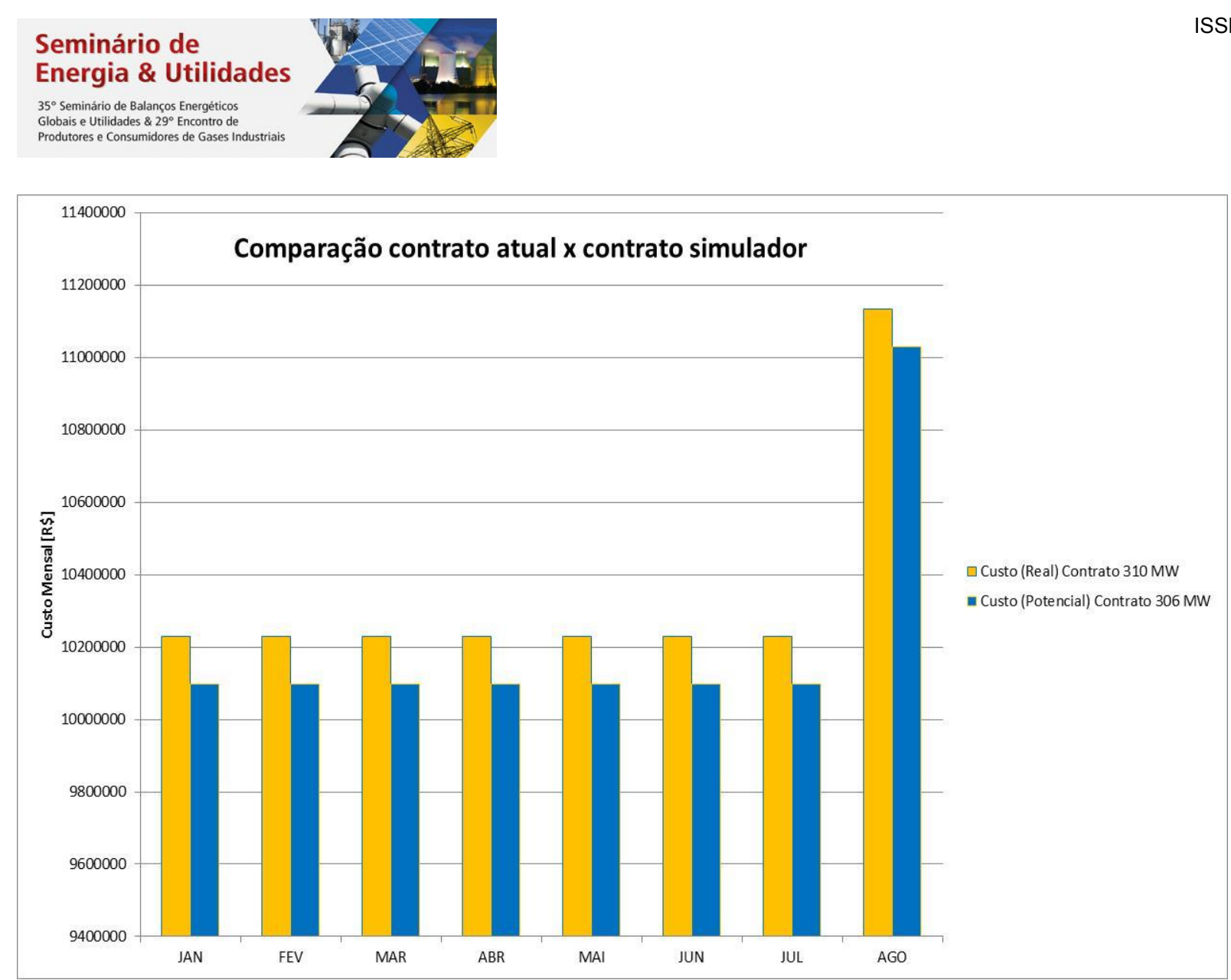

Figura 7 - Comparação entre o valor atual de contrato e o sugerido pelo simulador.

Analisando o gráfico comparativo observa-se que se o contrato tivesse sido concretizado em Janeiro de 2013 e com o valor sugerido pelo simulado (306 MW), até o presente momento haveria uma economia de $R \$ 1.028 .000,00$, com projeção (potencial) de economia de 1,5 milhões ao final de 2013.

\section{CONCLUSÃo}

O simulador mostrou-se eficaz para a redução do custo com demanda de energia elétrica na UPV (ganho potencial de 1,5 milhões em um ano), quando comparado com o contrato atual.

Torna-se necessária a revisão anual dos valores de contrato, de acordo com os resultados apresentados e com as particularidades de cada ano (programação de paradas da CTE-2, entrada de novas cargas na UPV, etc). Assim como é relevante salientar a importância do conhecimento / entendimento das resoluções da ANEEL pertinentes a demanda de energia elétrica.

Em suma, os objetivos do trabalho foram alcançados e uma nova ferramenta para auxílio na tomada de decisão encontra-se disponibilizada para a Gerência de Distribuição de Energéticos. 